\title{
Large-scale Boolean Matching
}

Hadi Katebi and Igor L. Markov

University of Michigan, 2260 Hayward St., Ann Arbor, MI 48109

\{hadik, imarkov\}@eecs.umich.edu

\section{Chapter Overview}

In this chapter, we propose a methodology for Boolean matching under permutations of inputs and outputs (PP-equivalence checking problem) - a key step in incremental logic design that identifies large sections of a netlist that are not affected by a change in specifications. When a design undergoes incremental changes, large parts of the circuit may remain unmodified. In these cases, the original and the slightly modified circuits share a number of functionally equivalent sub-circuits. Finding and reutilizing the equivalent subcircuits reduces the amount of work in each design iteration and accelerates design closure. In this chapter, we present a combination of fast and effective techniques that can efficiently solve the PPequivalence checking problem in practice. Our approach integrates graph-based, simulation-driven and SAT-based techniques to make Boolean matching feasible for large circuits. We verify the validity of our approach on ITC'99 benchmarks. The experimental results confirm scalability of our techniques to circuits with hundreds and even thousands of inputs and outputs.

The remainder of this chapter is organized as follows. Section 2 describes the motivation of this work. Section 3 provides relevant background and discusses previous work on Boolean matching. Section 4 gives an overview of proposed signaturebased techniques. Section 5 describes our SAT-based matching approach. Section 6 validates our method in experiments on available benchmarks, and Section 7 summarizes our work.

\section{Introduction}

Boolean matching is the problem of determining whether two Boolean functions are functionally equivalent under the permutation and negation of inputs and outputs. 
This formulation is usually referred to as the generalized Boolean matching problem or PNPN-equivalence checking (PNPN stands for Permutation and Negation of outputs and Permutation and Negation of inputs); however, different variants of the problem have been introduced for different synthesis and verification applications. The matching problem that we discuss in this chapter is PP-equivalence checking: two Boolean functions are called PP-equivalent if they are equivalent under permutation of inputs and permutation of outputs. The simplest method to determine whether two $n$-input $m$-output Boolean functions are PP-equivalent is to explicitly enumerate all the $m ! n$ ! possible matches and perform tautology checking on each. However, this exhaustive search is computationally intractable.

PP-equivalence checking finds numerous applications in verification and logic synthesis. In many cases, an existing design is modified incrementally leaving a great portion of the design untouched. In these cases, large isomorphic sub-circuits exist in original and slightly modified circuits [15]. Identifying such sub-circuits and reutilizing them whenever possible saves designers a great amount of money and time. Due to the fact that modifications to the original circuit are introduced by changing certain specifications and the fact that even a slight change in specifications can lead to large changes in implementation [9], PP-equivalence checking helps designers identify isomorphic and other equivalent sub-circuits.

Specifically, PP-equivalence checking can be used to find the minimal set of changes in logic, known as logic difference, between the original design and the design with modified specification. DeltaSyn [10] is a tool developed at IBM Research that identifies and reports this logic difference. The current version of DeltaSyn uses a relatively inefficient and unscalable Boolean matcher that only exploits the symmetry of inputs to prune the search space.

Incremental Sequential Equivalence Checking (SEC) is another application of PP-equivalence checking where isomorphic sub-circuits can be used to create a number of highly-likely candidate equivalent nodes [15]. The current implementation of incremental SEC tires to find isomorphic subgraphs by performing extended simulation and finding structural similarities. Although the Boolean approach presented in our paper does not fully exploit the structural similarities between two circuits, we believe that our techniques combined with structural verification techniques create a much more powerful tool for detecting isomorphic subgraphs.

Motivated by the practical importance of PP-equivalence checking in many EDA applications, we develop fast and scalable Boolean matching algorithms and implement them in the ABC package - an established system for synthesis and verification of combinational and sequential logic circuits [12]. The collection of all these techniques creates a powerful Boolean matching module that can be integrated into Combinational Equivalence Checking (CEC) to enhance its functionality. To this end, CEC requires two designs whose primary I/Os match by name. Our work allows one to relax this requirement with the help of a Boolean matcher. We call the new command Enhanced CEC (ECEC). Figure 1 shows how our Boolean matcher is integrated with CEC.

In general, algorithms for Boolean matching fall into two major categories: signature-based and canonical form based. A signature is a property of an input 

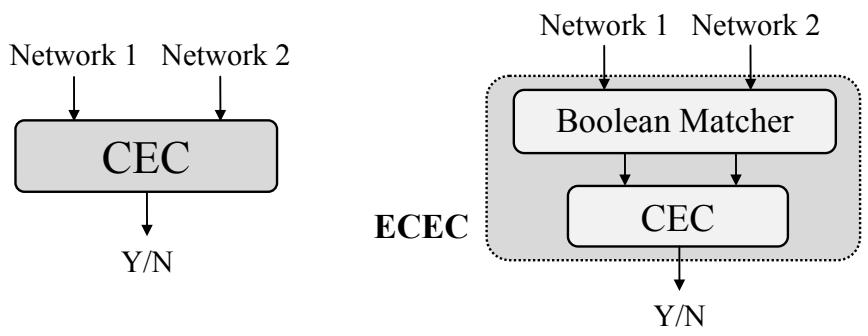

Fig. 1 The CEC (pre-existing) and ECEC (our) flows.

or an output that is invariant under permutations and negation of inputs. The goal of signature-based matching is to prune the Boolean matching space by filtering out impossible I/O correspondences $[4,1]$. On the other hand, in matching based on canonical forms, first canonical representations of two Boolean functions are computed and then compared against each other to find valid I/O matches [3, 2]. Here, our PP-equivalence checking method first prunes the search space using graph algorithms and simulation signatures, then it invokes SAT-solving until exact I/O matches are found (Figure 2).

Main contributions of our work include:

1. Analyzing functional dependency. In a Boolean network with multiple outputs, some inputs affect only a fraction of the outputs, and different outputs are affected in different ways. Hence, by analyzing the functional dependency of outputs on inputs, we can distinguish the I/Os.

2. Exploiting input observability and output controllability. We use the observability of inputs and the controllability of outputs as (1) effective matching signatures, and (2) ordering heuristics for our SAT-based matching.

3. Building a SAT-tree. When information about controllability, observability, and all simulation-based information are exhausted, we resort to SAT-solving and optimize the efficiency of SAT calls. This is accomplished through the concept of a SAT-tree, which is pruned in several ways.

4. Pruning SAT-tree using SAT counterexamples. In our SAT-based matching, the SAT-solver returns a counterexample whenever it finds an invalid match. The information in these counterexamples is then used to prune the SAT-tree.

Exponential space of possible matches

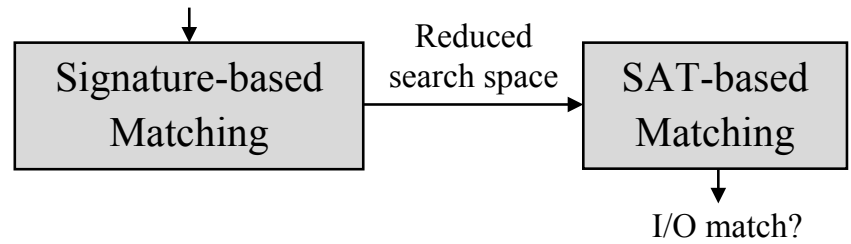

Fig. 2 Overview of our proposed Boolean matching approach. 


\section{Background and Previous Work}

In this section, we first review some common definitions and notation. Then, we explain the And-Inverter representation of Boolean networks and we compare its usage to that of conventional Binary Decision Diagrams. Next, we discuss Boolean satisfiabilty and explore its role in combinational equivalence checking. Relevant work in Boolean matching is reviewed at the end of this section.

\subsection{Definitions and Notation}

In the following definitions, an input set of a Boolean network $N$ refers to the set of all the inputs of $N$. Similarly, an output set of $N$ refers to the set of all the outputs of $N$. An I/O set is either an input set or an output set.

Definition 1. A partition $P_{x}=\left\{X_{1}, \ldots, X_{k}\right\}$ of an I/O set $X=\left\{x_{1}, \ldots, x_{n}\right\}$ is a collection of subsets $X_{1}, \ldots, X_{k}$ of $X$ such that $\cup_{i=1}^{k} X_{i}=X$ and $X_{i} \cap X_{j}=\emptyset$ for all $i \neq j$. Partition size of $P_{X}$ is the number of subsets in $P_{x}$ and is denoted by $\left|P_{x}\right|$. Each $X_{i}$ in $P_{x}$ is called an I/O cluster of $P_{x}$. The cardinality of $X_{i}$, denoted by $\left|X_{i}\right|$, is the number of I/Os in $X_{i}$.

Definition 2. A partition $P_{x}=\left\{X_{1}, \ldots, X_{k}\right\}$ of set $X$ is an ordered partition if the subsets $X_{1}, \ldots, X_{k}$ are totally ordered, i.e., for any two subsets $X_{i}$ and $X_{j}$, it is known whether $X_{i}<X_{j}$ or $X_{j}<X_{i}$.

Definition 3. Two ordered partitions $P_{x}=\left\{X_{1}, \ldots, X_{k}\right\}$ of set $X$ and $P_{y}=\left\{Y_{1}, \ldots, Y_{k}\right\}$ of set $Y$ are isomorphic if and only if $\left|P_{X}\right|=\left|P_{y}\right|=k$ and $\left|X_{i}\right|=\left|Y_{i}\right|$ for all $i$, and nonisomorphic otherwise. Two isomorphic partitions are called complete if and only if $\left|X_{i}\right|=\left|Y_{i}\right|=1$ for all $i$.

Definition 4. The positive cofactor of function $f\left(x_{1}, . ., x_{n}\right)$ with respect to variable $x_{i}$, denoted by $f_{x_{i}}$, is $f\left(x_{1}, . ., x_{i}=1, \ldots, x_{n}\right)$. Similarly, the negative cofactor of $f\left(x_{1}, . ., x_{n}\right)$ with respect to variable $x_{i}$, denoted by $f_{x_{i}^{\prime}}$, is $f\left(x_{1}, . ., x_{i}=0, \ldots, x_{n}\right)$.

Definition 5. A function $f\left(x_{1}, \ldots, x_{n}\right)$ is positive unate in variable $x_{i}$ if and only if the negative cofactor of $f$ with respect to $x_{i}$ is covered by the positive cofactor of $f$ with respect to $x_{i}$, i.e., $f_{x_{i}^{\prime}} \subseteq f_{x_{i}}$. Likewise, $f$ is negative unate in variable $x_{i}$ if and only if $f_{x_{i}} \subseteq f_{x_{i}^{\prime}} . f$ is called binate in $x_{i}$ if it is not unate in it.

\subsection{And-Inverter Graphs (AIGs)}

Recent tools for scalable logic synthesis, e.g., ABC, represent Boolean functions using the And-Inverter Graph (AIG) data structure. An AIG is a Boolean network 
composed of two-input AND gates and inverters. Structural hashing of an AIG is a transformation that reduces the AIG size by partially canonicalizing the AIG structure [13]. Representing a Boolean function in its AIG form is preferable to its $\mathrm{Bi}$ nary Decision Diagram (BDD) form mainly because AIGs result in smaller space complexity. Also, functional simulation can be performed much faster on AIGs, but AIGs are only locally canonical.

\subsection{Boolean Satisfiability and Equivalence-checking}

Boolean Satisfiability (SAT) is the problem of determining whether there exists a variable assignment to a Boolean formula that forces the entire formula evaluate to true; if such an assignment exists, the formula is said to be satisfiable and otherwise unsatisfiable. Pioneering techniques developed to solve the SAT problem were introduced by Davis, Putnam, Logemann and Loveland in early 1960s. They are now referred to as DPLL algorithm [7, 6]. Modern SAT solvers, such as MiniSAT [8], have augmented DPLL search by adding efficient conflict analysis, clause learning, back-jumping and watched literals to the basic concepts of DPLL.

SAT is studied in a variety of theoretical and practical contexts, including those arising in EDA. CEC is one of the main applications of SAT in EDA. If two singleoutput Boolean functions $f$ and $g$ are equivalent, then $f \oplus g$ must always evaluate to 0 , and vice versa. Now, instead of simulating all input combinations, we take advantage of SAT solvers: if $f \bigoplus g$ is unsatisfiable, then $f \bigoplus g$ is zero for all input combinations and hence $f$ and $g$ are equivalent; and if $f \bigoplus g$ is satisfiable, then $f$ and $g$ are not equivalent and the satisfying assignment found by the SAT-solver is returned as a counterexample. $f \bigoplus g$ is called the miter of $f$ and $g$ [14]. If $f$ and $g$ have more than one output, say $m$ outputs $f_{1}, \ldots, f_{m}$ and $g_{1}, \ldots, g_{m}, M_{i}=f_{i} \bigoplus g_{i}$ is first computed for all $i$ and then $M_{1}+\ldots+M_{m}$ is constructed as the miter of $f$ and $g$. In our approach, instead of building one miter for the entire circuit and handing it off to the SAT solver, we try to find equivalent intermediate signals by simulation, and use SAT to prove their equivalence. Counterexamples from SAT are used to refine simulation.

\subsection{Previous Work}

Research in Boolean matching started in the early 1980s with main focus on technology mapping (cell binding). A survey of Boolean matching techniques for library binding is given in [4]. Until recently, Boolean matching techniques scaled only to 10-20 inputs and one output $[5,2]$, which is sufficient for technology mapping, but not for applications considered in our work. In 2008, Abdollahi and Pedram presented algorithms based on canonical forms that can handle libraries with numerous cells limited to approximately 20 inputs [2]. Their approach uses generalized sig- 
natures (signatures of one or more variables) to find a canonicity-producing (CP) phase assignment and ordering for variables.

A DAC 2009 paper by Wang, Chan and Liu [17] offers simulation-driven and SAT-based algorithms for checking P-equivalence that scale beyond the needs of technology mapping. Since our proposed techniques also use simulation and SAT to solve the PP-equivalence checking problem, we should articulate the similarities and the differences. Firstly, we consider the more general problem of PP-equivalence checking where permutation of outputs (beside permutation of inputs) is allowed. In PP-equivalence, the construction of miters must be postponed until the outputs are matched, which seems difficult without matching the inputs. To address this challenge, we develop the concept of SAT-tree which is pruned to moderate the overall runtime of PP-matching. In addition to our SAT-based approach, we also use graph-based techniques in two different ways: to initially eliminate impossible I/O correspondences and to prune our SAT-tree. Furthermore, we have implemented three simulation types; two as signatures for outputs (type 1 and type 3 ) and one as a signature for inputs (type 2). While our type-2 simulation is loosely related to one of the techniques described in [17], the other two simulations are new. We additionally introduce effective heuristics that accelerate SAT-based matching.

\section{Signature-based Matching Techniques}

We now formalize the PP-equivalence checking problem and outline our Boolean matching approach for two $n$-input $m$-output Boolean networks.

Definition 6. Consider two I/O sets $X$ and $Y$ of two Boolean networks $N_{1}$ and $N_{2}$ with their two isomorphic ordered partitions $P_{x}=\left\{X_{1}, \ldots, X_{k}\right\}$ and $P_{y}=\left\{Y_{1}, \ldots, Y_{k}\right\}$. A cluster mapping of $X_{i}$ to $Y_{i}$, denoted by $X_{i} \mapsto Y_{i}$, is defined as the mapping of I/Os in $X_{i}$ to all possible permutations of I/Os in $Y_{i}$. A mapping of $X$ to $Y$ with respect to $P_{x}$ and $P_{y}$, denoted by $X \mapsto Y$, is defined as mapping of all same-index clusters of $X$ and $Y$, i.e., $X_{i} \mapsto Y_{i}$ for all $i . X \mapsto Y$ is called a complete mapping if $P_{x}$ and $P_{y}$ are complete.

Given two input sets $X$ and $Y$ and two outputs sets $Z$ and $W$ of two Boolean networks $N_{1}$ and $N_{2}$, the goal of PP-equivalence checking is to find two complete mappings $X \mapsto Y$ and $Z \mapsto W$ such that those mappings make $N_{1}$ and $N_{2}$ behave functionally the same. In order to accomplish this, we first partition or refine these I/O sets based on some total ordering criteria. This so-called signature-based matching allows us to identify and eliminate impossible I/O matches. After this phase, we rely on SAT-solving to find the two complete mappings. Furthermore, Definition 6 implies the following lemma.

Lemma 1. If at any point in the refinement process of two I/O sets $X$ and $Y, P_{x}$ and $P_{y}$ become non-isomorphic, we conclude that $N_{1}$ and $N_{2}$ behave differently and we stop the Boolean matching process. 
As mentioned earlier, refinement at each step requires a total ordering criterion, tailored to the specific refinement technique used. Therefore, whenever we introduce a new matching technique, we also explain its ordering criterion. Furthermore, the following techniques are applied to the two input circuits one after another.

\subsection{Computing I/O Support Variables}

Definition 7. Input $x$ is a support variable of output $z$ and output $z$ is a support variable of input $x$, if there exists an input vector $V$ such that flipping the value of $x$ in $V$ flips the value of $z$.

Definition 8. The support of input (or output) $x$, denoted by $\operatorname{Supp}(x)$, is the set of all the support variables of $x$. The cardinality of the support of $x$, denoted by $|\operatorname{Supp}(x)|$, is the number of I/Os in $\operatorname{Supp}(x)$. The degree of $x$, denoted by $D(x)$, is defined as the cardinality of its support.

The goal here is to find outputs that might be functionally affected by a particular input and inputs that might functionally affect a particular output. Here, we contrast functionally matching with structurally matching in the sense that two structurally different circuits with the same functionality should have the same I/O support. In general, the lack of structural dependency between an output and an input precludes a functional dependency, and the presence of a structural dependency most often indicates a functional dependency - this can usually be confirmed by random simulation, and in rare cases requires calling a SAT-solver [11].

Example 1. Consider a 4-bit adder with input set $X=\left\{C_{i n}, A_{0}, \ldots, A_{3}, B_{0}, \ldots, B_{3}\right\}$ and output set $Z=\left\{S_{0}, \ldots, S_{4}\right\}$. The ripple-carry realization of this adder is shown in Figure 3.

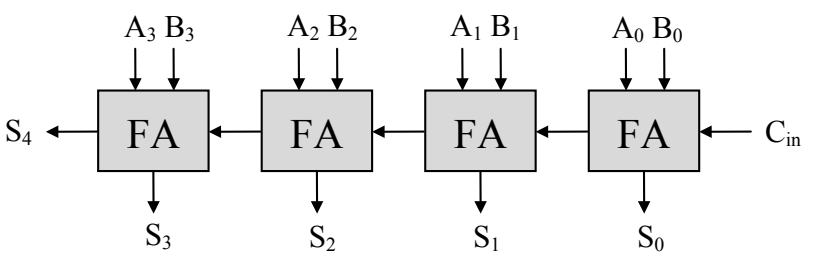

Fig. 3 4-bit ripple-carry adder.

It is evident from the above circuit that $A_{0}$ can affect the values of $S_{0}, \ldots, S_{4}$ and $A_{1}$ can affect the value of $S_{1}, \ldots, S_{4}$. Hence, $\operatorname{Supp}\left(A_{0}\right)=\left\{S_{0}, \ldots, S_{4}\right\}$ and $\operatorname{Supp}\left(A_{1}\right)=$ $\left\{S_{1}, \ldots, S_{4}\right\}$. Similarly, the value of $S_{0}$ is only affected by the value of $A_{0}, B_{0}$ and $C_{i n}$. Hence, $\operatorname{Supp}\left(S_{0}\right)=\left\{A_{0}, B_{0}, C_{i n}\right\}$. 


\subsection{Initial refinement of I/O clusters}

Lemma 2. Two inputs (outputs) can match only if they have the same degree.

Taking advantage of Lemma 2, we can initially refine the I/O sets by gathering all I/Os of the same degree in one subcluster and then sort the subclusters based on the following ordering criterion:

Ordering criterion 1. Let $i$ and $j$ be two inputs (outputs) with different degrees and assume that $D(i)<D(j)$. Then, the subcluster containing $i$ precedes the subcluster containing $j$.

Example 2. Consider the 4-bit adder of Example 1. The degree of each input and output is given below:

$$
\begin{aligned}
& D\left(A_{0}\right)=D\left(B_{0}\right)=D\left(C_{\text {in }}\right)=5 \\
& D\left(A_{1}\right)=D\left(B_{1}\right)=4 \\
& D\left(A_{2}\right)=D\left(B_{2}\right)=3 \\
& D\left(A_{3}\right)=D\left(B_{3}\right)=2 \\
& D\left(S_{0}\right)=3 \\
& D\left(S_{1}\right)=5 \\
& D\left(S_{2}\right)=7 \\
& D\left(S_{3}\right)=D\left(S_{4}\right)=9
\end{aligned}
$$

The ordered partitions of the I/O sets of the 4-bit adder after initial refinement are: $P_{x}=\left\{\left\{A_{3}, B_{3}\right\},\left\{A_{2}, B_{2}\right\},\left\{A_{1}, B_{1}\right\},\left\{A_{0}, B_{0}, C_{i n}\right\}\right\}$ $P_{z}=\left\{\left\{S_{0}\right\},\left\{S_{1}\right\},\left\{S_{2}\right\},\left\{S_{3}, S_{4}\right\}\right\}$

\subsection{Refining Outputs by Minterm Count}

Lemma 3. Two outputs can match only if their Boolean functions have the same number of minterms.

Ordering criterion 2. Let $i$ and $j$ be two outputs in the same output cluster and let $M(i)$ and $M(j)$ be the number of minterms of $i$ and $j$, respectively. If $M(i)<M(j)$, then the subcluster containing $i$ is smaller than the subcluster containing $j$.

Minterm count is another effective output signature which is only practical when the circuit is represented in BDD form. In fact, the widely adopted way to count the minterms of a Boolean network represented in AIG is to first convert it to a BDD, but this approach is limited in scalability [16].

\subsection{Refining I/O by Unateness}

Lemma 4. Two outputs match only if they are unate in the same number of inputs. Similarly, two inputs match only if the same number of outputs is unate in them. 
Ordering criterion 3. Let $i$ and $j$ be two outputs in the same output cluster. Assume that $U$ nate $(i)$ and $U$ nate $(j)$ are the number of unate variables of $i$ and $j$ respectively, and let Unate $(i)<\operatorname{Unate}(j)$. Then, the output subcluster containing $i$ is smaller than the subcluster containing $j$. Similarly, let $i$ and $j$ be two inputs in one input cluster. Assume that $U$ nate $(i)$ and $\operatorname{Unate}(j)$ are the number of outputs that are unate in $i$ and $j$ respectively, and let $U$ nate $(i)<\operatorname{Unate}(j)$. Then, the input subcluster containing $i$ is smaller than the subcluster containing $j$.

Although unateness generates powerful signatures for Boolean matching, computing unateness in an AIG encounters the same limitation as was discussed for counting the number of minterms. Hence, refinement based on unateness is only practical for small Boolean networks.

\subsection{Scalable I/O Refinement by Dependency Analysis}

We mentioned earlier that the degree of each I/O is an effective signature for initial refinement of I/O sets. Here, we generalize this concept by not only considering the number of support variables but also carefully analyzing I/O dependencies.

Definition 9. Let $x$ be an input (output) and let $\operatorname{Supp}(x)=\left\{z_{1}, \ldots, z_{k}\right\}$. We define a sequence $S=\left(s_{1}, \ldots, s_{k}\right)$ of unsigned integers where each $s_{i}$ is the index of the output (input) cluster that $z_{i}$ belongs to. After sorting $S$, we call it support signature of $x$ and we denote it by $\operatorname{Sign}(x)$.

Lemma 5. Two I/Os $i$ and $j$ in the same I/O cluster are distinguishable if Sign $(i) \neq$ $\operatorname{Sign}(j)$.

Ordering criterion 4. Let $i$ and $j$ be two I/Os in the same I/O cluster. Assume that $\operatorname{Sign}(i)<\operatorname{Sign}(j)$ meaning that the support signature of $i$ is lexicographically smaller than the support signature of $j$. Then, the subcluster containing $i$ precedes the subcluster containing $j$.

Example 3. Consider a circuit with input set $X=\left\{x_{1}, x_{2}, x_{3}\right\}$ and output set $Z=$ $\left\{z_{1}, z_{2}, z_{3}\right\}$ where $z_{1}=\overline{x_{1}}, z_{2}=x_{1} \cdot x_{2}$ and $z_{3}=\overline{x_{2} \cdot x_{3}}$. The I/O supports of the circuit are: $\operatorname{Supp}\left(z_{1}\right)=\left\{x_{1}\right\}, \operatorname{Supp}\left(z_{2}\right)=\left\{x_{1}, x_{2}\right\}, \operatorname{Supp}\left(z_{3}\right)=\left\{x_{2}, x_{3}\right\}$ and $\operatorname{Supp}\left(x_{1}\right)=$ $\left\{z_{1}, z_{2}\right\}, \operatorname{Supp}\left(x_{2}\right)=\left\{z_{2}, z_{3}\right\}, \operatorname{Supp}\left(x_{3}\right)=\left\{z_{3}\right\}$. Since $D\left(z_{1}\right)=1$ and $D\left(z_{2}\right)=$ $D\left(z_{3}\right)=2$, and $D\left(x_{3}\right)=1$ and $D\left(x_{1}\right)=D\left(x_{2}\right)=2$, we can initialize I/O clusters as follows: $P_{z}=\left\{\left\{z_{1}\right\},\left\{z_{2}, z_{3}\right\}\right\}, P_{x}=\left\{\left\{x_{3}\right\},\left\{x_{1}, x_{2}\right\}\right\}$. Now, we try refining based on support signatures. The signatures for $z_{1}, z_{3}, x_{1}$ and $x_{2}$ are: $\operatorname{Sign}\left(z_{2}\right)=(2,2)$, $\operatorname{Sign}\left(z_{3}\right)=(1,2), \operatorname{Sign}\left(x_{1}\right)=(1,2), \operatorname{Sign}\left(x_{2}\right)=(2,2) . \operatorname{Since} \operatorname{Sign}\left(z_{3}\right)<\operatorname{Sign}\left(z_{2}\right)$ and $\operatorname{Sign}\left(x_{1}\right)<\operatorname{Sign}\left(x_{2}\right)$, we can further partition $\left\{z_{2}, z_{3}\right\}$ and $\left\{x_{1}, x_{2}\right\}$, hence $P_{z}=\left\{\left\{z_{1}\right\},\left\{z_{3}\right\},\left\{z_{2}\right\}\right\}$ and $P_{x}=\left\{\left\{x_{3}\right\},\left\{x_{1}\right\},\left\{x_{2}\right\}\right\}$.

After each round of refinement based on I/O dependencies, we check if any I/O cluster is further partitioned. If a new partition is added, the algorithm performs another round of refinement. The procedure terminates when no new refinement occurs after a certain number of iterations. 


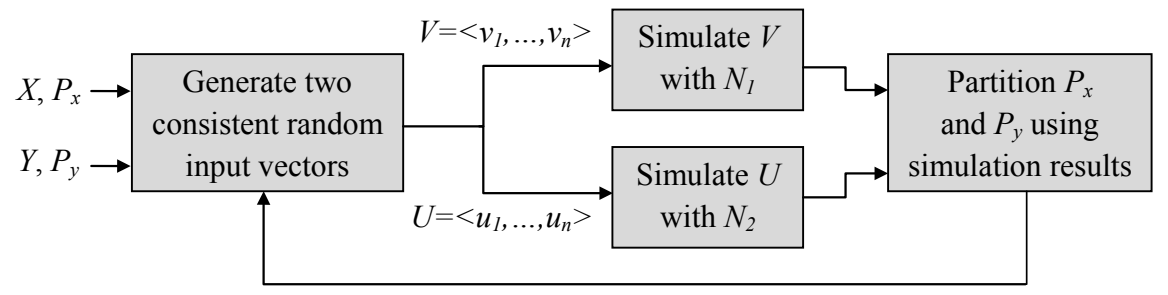

Fig. 4 Flow of the proposed I/O refinement by random simulation.

\subsection{Scalable I/O Refinement by Random Simulation}

Functional simulation holds the promise to quickly prune away unpromising branches of search, but this seems to require a matching of outputs. Instead, we find pairs of input vectors that sensitize comparable functional properties of the two circuits. Let $V=\left\langle v_{1}, \ldots, v_{n}\right\rangle$ be an input vector of Boolean network $N$. The result of simulating $V$ on $N$ is called the output vector of $N$ under $V$ and is denoted by $R_{v}=<r_{1}, \ldots, r_{m}>$.

Definition 10. Let $N$ be a Boolean network with input set $X$ and let $P_{x}=\left\{X_{1}, \ldots, X_{k}\right\}$ be an ordered partition of $X$. An input vector $V=\left\langle v_{1}, \ldots, v_{n}\right\rangle$ is said to be proper if it assigns the same value ( 0 or 1$)$ to all the inputs of $N$ which are in the same input cluster, i.e., $v_{i}=v_{j}$ if $i, j \in X_{l}$ for some $l$. The input vectors consisting of all 0 s or all $1 \mathrm{~s}$ are the trivial proper input vectors.

Definition 11. Let $X=\left\{x_{1}, \ldots, x_{n}\right\}$ and $Y=\left\{y_{1}, \ldots, y_{n}\right\}$ be the input sets of two Boolean networks $N_{1}$ and $N_{2}$ and let $P_{x}=\left\{X_{1}, \ldots, X_{k}\right\}$ and $P_{y}=\left\{Y_{1}, \ldots, Y_{k}\right\}$ be two ordered partitions defined on them. Two proper random input vectors $V=\left\langle v_{1}, \ldots, v_{n}\right\rangle$ and $U=<u_{1}, \ldots, u_{n}>$ of $N_{1}$ and $N_{2}$ are said to be consistent if, for all $1 \leq l \leq k$, $x_{i} \in X_{l}$ and $y_{j} \in Y_{l}$ imply that $v_{i}=u_{j}$.

Intuitively, two consistent random input vectors try to assign the same value to all potentially matchable inputs of the two Boolean networks. In the next three subsections, we distinguish three types of simulation based on pairs of consistent random input vectors that help us sensitize certain functional properties of the two circuits. The flow of the I/O refinement by random simulation is shown in Figure 4.

\subsubsection{Simulation type 1}

Lemma 6. Let $V$ be a proper random input vector and let $R_{v}=<r_{1}, \ldots, r_{m}>$ be the corresponding output vector under $V$. Two outputs $i$ and $j$ in one output cluster are distinguishable if $r_{i} \neq r_{j}$.

The above lemma classifies outputs based on their values ( 0 or 1$)$ using the following ordering criterion.

Ordering criterion 5. The output subcluster of all 0s precedes the output subcluster of all $1 \mathrm{~s}$. 


\subsubsection{Simulation type 2}

Definition 12. Let $V$ be a proper random input vector and let $R_{v}=\left\langle r_{1}, \ldots, r_{m}\right\rangle$ be the corresponding output vector under $V$. Let $V^{\prime}$ be another input vector created by flipping the value of input $x$ in $V$ and let $R_{v^{\prime}}=\left\langle r_{1}^{\prime}, \ldots, r_{m}^{\prime}\right\rangle$ be the corresponding output vector under $V^{\prime}$. The observability of input $x$ with respect to $V$ denoted by $\operatorname{Obs}(x)$ is defined as the number of flips in the outputs caused by $V^{\prime}$, i.e., the number of times $r_{i} \neq r_{i}^{\prime}$.

Lemma 7. Two inputs $i$ and $j$ in one input cluster are distinguishable if $\operatorname{Obs}(i) \neq$ $\operatorname{Obs}(j)$.

Ordering criterion 6. Let $i$ and $j$ be two inputs in one input cluster and let $O b s(i)<$ $\operatorname{Obs}(j)$. Then, the input subcluster containing $i$ precedes the input subcluster containing $j$.

\subsubsection{Simulation type 3}

Definition 13. Consider a proper random input vector $V$ and its corresponding output vector $\left.R_{v}=<r_{1}, \ldots, r_{m}\right\rangle$. Let $V_{1}, \ldots, V_{n}$ be $n$ input vectors where vector $V_{i}$ is created by flipping the value of $r_{i}$ in $V$. Also, let $R_{v_{1}}=<r_{1,1}, \ldots, r_{1, m}>, \ldots, R_{v_{n}}=<$ $r_{n, 1}, \ldots, r_{, m}>$ be the corresponding output vectors under $V_{1}, \ldots, V_{n}$. The controllability of output $z$ with respect to $V$ denoted by $\operatorname{Ctrl}(z)$ is defined as the number of times $r_{i} \neq r_{j, i}$, for all $1 \leq j \leq n$.

Lemma 8. Two outputs $i$ and $j$ in one output cluster are distinguishable if Ctrl $(i) \neq$ $\operatorname{Ctrl}(j)$.

Ordering criterion 7. Let $i$ and $j$ be two outputs in one output cluster and let $\operatorname{Ctrl}(i)<\operatorname{Ctrl}(j)$. Then, the output subcluster containing $i$ precedes the output subcluster containing $j$.

Example 4. Consider an 8-to-1 multiplexer with input set $X=\left\{a_{0}, \ldots, a_{7}, s_{0}, s_{1}, s_{2}\right\}$ and output $z$ where $a_{0}, \ldots, a_{7}$ denote data signals and $s_{0}, s_{1}, s_{2}$ are control signals. Initially $P_{x}$ has only one partition, namely $X$. Initial refinement and refinement by dependency analysis do not partition $P_{x}$, hence we try random simulation. Here, we can only use type 2 simulation since simulation of type 1 and 3 are for refining output clusters. First, we consider the trivial input vector $V$ of all 0 s. We flip one input in $V$ at a time and we apply the resulting vectors to the multiplexer. Only flipping $a_{0}$ flips $z$; hence, $P_{x}=\left\{\left\{a_{1}, \ldots, a_{7}, s_{0}, s_{1}, s_{2}\right\},\left\{a_{0}\right\}\right\}$. Then we try the trivial input vector $V$ of all $1 \mathrm{~s}$. This time flipping $a_{7}$ flips $z$; hence, $P_{x}=\left\{\left\{a_{1}, \ldots, a_{6}, s_{0}, s_{1}, s_{2}\right\},\left\{a_{7}\right\},\left\{a_{0}\right\}\right\}$. Next, we put $a_{0}$ to 1 and all the other inputs to 0 . Now flipping $s_{0}, s_{1}, s_{2}$ flips $z$, hence $P_{x}=\left\{\left\{a_{1}, \ldots, a_{6}\right\},\left\{s_{0}, s_{1}, s_{2}\right\},\left\{a_{7}\right\},\left\{a_{0}\right\}\right\}$. If we continue partitioning based on the remaining proper input vectors no additional refinement will be gained.

After matching I/Os using random simulation, we check if any progress is achieved in refining I/O clusters. If a new cluster is added, the algorithm continues 
refining based on random simulation. The procedure terminates when no new refinement occurs in input or output subclusters after a certain number of iterations. Here, the number of iterations does not affect the correctness of the algorithm. However, too few iterations might diminish the impact of matching by random simulation, and excessive iterations offer no improvement. Our current implementation limits iterations to 200 .

\section{SAT-based Search}

The scalable methods we introduced so far typically reduce the number of possible matches from $n ! m$ ! to hundreds or less, often making exhaustive search (with SATbased equivalence-checking) practical. However, this phase of Boolean matching can be significantly improved, and the techniques we develop facilitate scaling to even larger instances.

\subsection{SAT-based Input Matching}

The basic idea in our SAT-based matching approach is to build a tree data structure called SAT-tree that matches one input at a time from the remaining non-singleton input clusters. Subsequently, after an input is matched, all the outputs in its support which are not matched so far are also matched, one by one. In other words, we build a dual-purpose tree that repeatedly matches inputs and outputs until exact I/O matches are found. We take advantage of the following lemma to build our SAT-tree:

Lemma 9. Assume that two Boolean networks $N_{1}$ and $N_{2}$ with input sets $X=$ $\left\{x_{1}, \ldots, x_{n}\right\}$ and $Y=\left\{y_{1}, \ldots, y_{n}\right\}$ are functionally equivalent under two complete ordered partitions $P_{x}=\left\{X_{1}, \ldots, X_{n}\right\}$ and $P_{y}=\left\{Y_{1}, \ldots, Y_{n}\right\}$. Also, assume that $X_{l}=\left\{x_{i}\right\}$ and $Y_{l}=\left\{y_{j}\right\}$. Let $N_{1}^{\prime}$ be the positive (negative) cofactor of $N_{1}$ with respect to $x_{i}$ and $N_{2}^{\prime}$ be the positive (negative) cofactor of $N_{2}$ with respect to $y_{j} . N_{1}^{\prime}$ and $N_{2}^{\prime}$ with input sets $X^{\prime}=X-\left\{x_{i}\right\}$ and $Y^{\prime}=Y-\left\{y_{j}\right\}$ behave functionally the same under two complete ordered partitions $P_{x^{\prime}}=P_{x}-\left\{X_{l}\right\}$ and $P_{y^{\prime}}=P_{y}-\left\{Y_{l}\right\}$.

The inputs to the SAT-based matching algorithm are two ordered input partitions and two ordered output partitions. Here, we assume that some of the partitions are incomplete because if all partitions are complete, an exact match is already found. Without loss of generality, assume that in two ordered partitions $P_{x}=\left\{X_{1}, \ldots, X_{k}\right\}$ and $P_{y}=\left\{Y_{1}, \ldots, Y_{k}\right\}$ of sets $X$ and $Y, X_{1}, \ldots, X_{l-1}$ and $Y_{1}, \ldots, Y_{l-1}$ are all singleton clusters and $X_{l}, \ldots, X_{k}$ and $Y_{l}, \ldots, Y_{k}$ are non-singleton clusters. Repeatedly applying Lemma 9 allows us to create two new Boolean networks $N_{1}^{\prime}$ and $N_{2}^{\prime}$ by setting all the inputs in $X_{l}, \ldots, X_{k}$ and $Y_{l}, \ldots, Y_{k}$ to either constant 0 or constant 1 . In other words, we shrink input sets $X$ to $X^{\prime}=X-\left\{x \mid x \in\left\{X_{l}, \ldots, X_{k}\right\}\right\}$ and input set $Y$ to $Y^{\prime}=Y-\{y \mid y \in$ $\left.\left\{Y_{l}, \ldots, Y_{k}\right\}\right\}$ such that $X^{\prime}$ and $Y^{\prime}$ only contain the inputs that have exact match in $N_{1}$ 
and $N_{2}$. Note that, by definition, the ordered partitions $P_{x}^{\prime}=P_{x}-\left\{X_{l}, \ldots, X_{k}\right\}$ and $P_{y}^{\prime}=P_{y}-\left\{Y_{l}, \ldots, Y_{k}\right\}$ are complete partitions of $X^{\prime}$ and $Y^{\prime}$. According to Lemma 9 , $N_{1}^{\prime}$ and $N_{2}^{\prime}$ must be functionally equivalent if $N_{1}$ and $N_{2}$ are equivalent. $N_{1}^{\prime}$ and $N_{2}^{\prime}$ are called the Smallest Matching Sub-circuits (SMS) of $N_{1}$ and $N_{2}$.

After finding the SMS of $N_{1}$ and $N_{2}$, we try to expand $X^{\prime}$ and $Y^{\prime}$ back to $X$ and $Y$ by matching one input at a time. Let $X_{l}$ and $Y_{l}$ be the first two non-singleton input clusters of $P_{x}$ and $P_{y}$ and let $x_{i} \in X_{l}$. The goal here is to match $x_{i}$ with one of the $\left|Y_{l}\right|$ inputs in $Y_{l}$. Assume that $y_{j} \in Y_{l}$, and we pick $y_{j}$ as the first candidate to match $x_{i}$. Now, in order to reflect our matching decision, we partition $X_{l}$ and $Y_{l}$ to make $\left\{x_{i}\right\}$ and $\left\{y_{j}\right\}$ two singleton clusters; hence, $X_{l}$ is partitioned to $X_{l, 1}=\left\{x_{i}\right\}$ and $X_{l, 2}=X_{l}-\left\{x_{i}\right\}$ and $Y_{l}$ is partitioned to $Y_{l, 1}=\left\{y_{j}\right\}$ and $Y_{l, 2}=Y_{l}-\left\{y_{j}\right\}$. Complying with our previous notation, now $X_{l, 2}, \ldots, X_{k}$ and $Y_{l, 2}, \ldots, Y_{k}$ are the new non-singleton clusters. We then build two Boolean networks $N_{1}^{\prime \prime}$ and $N_{2}^{\prime \prime}$ from $N_{1}$ and $N_{2}$ by setting all the inputs in non-singleton clusters to either constant 0 or constant 1 , and we pass the miter of $N_{1}^{\prime \prime}$ and $N_{2}^{\prime \prime}$ to the SAT-solver. The SAT-solver may return either satisfiable or unsatisfiable. If the result is:

- unsatisfiable: $N_{1}^{\prime \prime}$ and $N_{2}^{\prime \prime}$ are functionally equivalent. In other words, $x_{i}$ and $y_{j}$ has been a valid match so far. Hence, first try to match the outputs in the supports of $x_{i}$ and $y_{j}$ (only the outputs that have not been matched so far) and then match the next two unmatched inputs in $X_{l, 2}$ and $Y_{l, 2}$.

- satisfiable: $N_{1}^{\prime \prime}$ and $N_{2}^{\prime \prime}$ are not functionally equivalent. In other words, $x_{i}$ cannot match $y_{j}$. Backtrack one level up and use the counterexample to prune the SATtree.

In a case where the SAT-solver times out, we terminate the matching process, and only report the I/Os matched by our signature-based techniques. Unlike early prototypes, our most optimized implementation does not experience this situation on the testcases used in our experiments.

\subsection{Pruning Invalid Input Matches By SAT Counterexamples}

Pruning the SAT-tree using counterexamples produced by SAT is a key step in our Boolean matching methodology. Continuing the scenario in Section 5.1, assume that the miter of $N_{1}^{\prime \prime}$ and $N_{2}^{\prime \prime}$ is satisfiable. Suppose that the SAT-solver returns an input vector $V=<v_{1}, \ldots, v_{l+1}>$ as the satisfying assignment. This input vector carries a crucial piece of information: the matching attempt before matching $x_{i}$ and $y_{j}$ was a successful match; otherwise we would have backtracked in the previous level and we would have never tried matching $x_{i}$ and $y_{j}$. Thus, the input vector $V$ sensitizes a path from $x_{i}$ and $y_{j}$ to the outputs of the miter.

According to Lemma 9, repeatedly computing negative and positive cofactors of $N_{1}$ and $N_{2}$ with respect to the values of $v_{1}, \ldots, v_{l}$ in $V$ results in two new Boolean networks $\hat{N}_{1}$ and $\hat{N}_{2}$ that must be functionally equivalent under some ordered partition $P_{x}-\left\{X_{1}, \ldots, X_{l}\right\}$ and $P_{y}-\left\{Y_{1}, \ldots, Y_{l}\right\}$. In other words, $\hat{N}_{1}$ and $\hat{N}_{2}$ are two smaller 
Boolean networks that only contain the inputs of $N_{1}$ and $N_{2}$ that have not found exact match so far. Since $\hat{N}_{1}$ and $\hat{N}_{2}$ are computed with respect to the values of $v_{1}, \ldots, v_{l}$ in $V$ and since $V$ is a vector that sensitizes a path form $x_{i}$ and $y_{j}$ to the output of the miter, we conclude that there exists an output in $\hat{N}_{1}$ that is functionally dependent on $x_{i}$. The existence of such an output ensures that $D\left(x_{i}\right)>0$. We can now apply our simple filtering signature from Lemma 2 to prune the SAT-tree. Specifically, $x_{i} \in X_{l}$ can match to $y_{q} \in Y_{l}(q \neq j)$ only if $D\left(x_{i}\right)=D\left(y_{q}\right)$ in $\hat{N}_{1}$ and $\hat{N}_{2}$.

Example 5. Consider two 8-to-1 multiplexers with outputs $z$ and $z^{\prime}$ and input sets $X=\left\{a_{0}, \ldots, a_{7}, s_{0}, s_{1}, s_{2}\right\}$ and $X^{\prime}=\left\{a_{0}^{\prime}, \ldots, a_{7}^{\prime}, s_{0}^{\prime}, s_{1}^{\prime}, s_{2}^{\prime}\right\}$. Refining $X$ and $X^{\prime}$ based on the techniques explained in Section 4 would result in two ordered partitions $P_{x}=$ $\left\{\left\{a_{1}, \ldots, a_{6}\right\},\left\{s_{0}, s_{1}, s_{2}\right\},\left\{a_{7}\right\},\left\{a_{0}\right\}\right\}$ and $P_{x^{\prime}}=\left\{\left\{a_{1}^{\prime}, \ldots, a_{6}^{\prime}\right\},\left\{s_{0}^{\prime}, s_{1}^{\prime}, s_{2}^{\prime}\right\},\left\{a_{7}^{\prime}\right\},\left\{a_{0}^{\prime}\right\}\right\}$ (refer to Example 4). In order to find exact input matches, we build our SATtree and we first try matching $s_{2}$ and $s_{0}^{\prime}$. The SAT-solver confirms the validity of this match. Then, $s_{1}$ matches $s_{1}^{\prime}$ and $s_{0}$ matches $s_{2}^{\prime}$. These two matches are also valid. So far, $P_{x}=\left\{\left\{a_{1}, \ldots, a_{6}\right\},\left\{s_{2}\right\},\left\{s_{1}\right\},\left\{s_{0}\right\},\left\{a_{7}\right\},\left\{a_{0}\right\}\right\}$ and $P_{x^{\prime}}=$ $\left\{\left\{a_{1}^{\prime}, \ldots, a_{6}^{\prime}\right\},\left\{s_{0}^{\prime}\right\},\left\{s_{1}^{\prime}\right\},\left\{s_{2}^{\prime}\right\},\left\{a_{7}^{\prime}\right\},\left\{a_{0}^{\prime}\right\}\right\}$. Now, we look at the next non-singleton input cluster and we match $a_{1}$ and $a_{1}^{\prime}$. Our SAT-solver specifies that matching $a_{1}$ and $a_{1}^{\prime}$ do not form a valid match and it returns vector $V$ in which $s_{0}^{\prime}=s_{2}=0, s_{1}^{\prime}=s_{1}=0$, $s_{2}^{\prime}=s_{0}=1, a_{7}^{\prime}=a_{7}=0, a_{0}^{\prime}=a_{0}=0, a_{1}^{\prime}=a_{1}=1$ as a counterexample. In order to see why $V$ is a counterexample of matching $a_{1}$ and $a_{1}^{\prime}$, we look at the cofactors of the two multiplexers, $c$ and $c^{\prime}$, where all the inputs in non-singleton clusters are set to 0: $c=a_{0} \overline{s_{2}} \overline{s_{1}} \overline{s_{0}}+a_{1} \overline{s_{2}} \overline{s_{1}} s_{0}+a_{7} s_{2} s_{1} s_{0}$ and $c^{\prime}=a_{0}^{\prime} \overline{s_{0}^{\prime}} \overline{s_{1}^{\prime}} \overline{s_{2}^{\prime}}+a_{1}^{\prime} \overline{s_{0}^{\prime}} \overline{s_{1}^{\prime}} s_{2}^{\prime}+a_{7}^{\prime} s_{0}^{\prime} s_{1}^{\prime} s_{2}^{\prime}$. Applying $V$ to $c$ and $c^{\prime}$ would result in $c=1$ and $c^{\prime}=0$. Since we know that $a_{1}$ does not match $a_{1}^{\prime}$, we use the counterexample to prune the SAT-tree. Specifically, we compute cofactors of the two multiplexers, $d$ and $d^{\prime}$, with respect to the values of matched inputs in $V$. So, $d=\overline{a_{1}} \overline{s_{2}} \overline{s_{1}} s_{0}$ and $d^{\prime}=a_{4}^{\prime} \overline{s_{0}^{\prime}} \overline{s_{1}^{\prime}} s_{2}^{\prime}$. In $d$ and $d^{\prime}, D\left(a_{1}\right)=D\left(a_{4}^{\prime}\right)=1$. This means that $a_{1}$ can only match $a_{4}^{\prime}$. In other words, we have pruned SAT search space by not matching $a_{1}$ to any of inputs $a_{2}^{\prime}, a_{3}^{\prime}, a_{5}^{\prime}$ and $a_{6}^{\prime}$. We continue matching inputs until we find valid matches.

\subsection{SAT-based Output Matching}

Let $Z$ and $W$ be the output sets of two Boolean networks $N_{1}$ and $N_{2}$ and let $P_{z}=\left\{Z_{1}, \ldots, Z_{k}\right\}$ and $P_{w}=\left\{W_{1}, \ldots, W_{k}\right\}$ be two ordered partitions defined on them. Continuing the scenario in Section 5.1, assume that $z_{i} \in Z_{l}$ is a support variable of $x_{i}, w_{j} \in W_{l}$ is a support variable of $y_{j}$, and $Z_{l}$ and $W_{l}$ are two non-singleton output clusters of $P_{z}$ and $P_{w}$. In order to verify if $z_{i}$ and $w_{j}$ match under current input correspondence, we add $z_{i} \oplus w_{j}$ to the current miter of $N_{1}^{\prime \prime}$ and $N_{2}^{\prime \prime}$ and we call SAT-solver once again. If SAT returns unsatisfiable, i.e., $z_{i}$ matches $w_{j}$, we continue matching the remaining unmatched outputs in the support of $x_{i}$ and $y_{j}$. If the result is satisfiable, we once again use the counterexample returned by SAT to prune the search space. 
Example 6. Consider two circuits $N_{1}$ and $N_{2}$ with input sets $X=\left\{x_{0}, \ldots, x_{3}\right\}$ and $Y=\left\{y_{0}, \ldots, y_{3}\right\}$, and output sets $Z=\left\{z_{0}, z_{1}\right\}$ and $W=\left\{w_{0}, w_{1}\right\}$ where $z_{0}=x_{0} \cdot x_{1}$. $\overline{x_{2}} \cdot \overline{x_{3}}, z_{1}=\overline{x_{0}} \cdot \overline{x_{1}} \cdot x_{2} \cdot x_{3}, w_{0}=\overline{y_{0}} \cdot \overline{y_{1}} \cdot y_{2} \cdot y_{3}$, and $w_{1}=y_{0} \cdot y_{1} \cdot \overline{y_{2}} \cdot \overline{y_{3}}$. For these two circuits, signature-based matching (discussed in Section 4) cannot distinguish any I/Os. Hence, we resort to SAT-solving. Assume that SAT search starts by matching $x_{0}$ to $y_{0}$. Since $\left\{z_{0}, z_{1}\right\} \in \operatorname{Supp}\left(x_{0}\right)$ and $\left\{w_{0}, w_{1}\right\} \in \operatorname{Supp}\left(y_{0}\right)$, the outputs of the circuits must be matched next. Among all valid matches, our SAT-solver can match $z_{0}$ to $w_{1}$ and $z_{1}$ to $w_{0}$. For the remaining space of the unmatched inputs, our SATsolver can validly match $x_{1}$ to $y_{1}, x_{2}$ to $y_{3}$, and $x_{3}$ to $y_{2}$, and finish the search.

\subsection{Pruning Invalid Output Matches By SAT Counterexamples}

When output $z_{i} \in Z_{l}$ does not match output $w_{j} \in W_{l}$, the counterexample returned by SAT is a vector $V$ that makes $z_{i}=1$ and $w_{j}=0$ or vice versa. This means that $z_{i}$ matches output $w_{q} \in W_{l}(q \neq j)$ only if $z_{i}=w_{q}$ under $V$. This simple fact allows us to drastically prune our SAT-tree whenever an invalid output match occurs.

\subsection{Pruning Invalid I/O Matches Using Support Signatures}

We demonstrated in Section 4.5 that support signatures of inputs and outputs can be used to refine I/O subclusters of a Boolean network. In this section, we show that support signatures can also be used in our SAT-tree to eliminate impossible I/O correspondences.

Lemma 10. Suppose that $x_{i} \in X_{l}$ and $y_{j} \in Y_{l}$ are two unmatched inputs of $N_{1}$ and $N_{2}$. Then, $x_{i}$ can match $y_{j}$ only if Sign $\left(x_{i}\right)=\operatorname{Sign}\left(y_{j}\right)$. Likewise, suppose that $z_{i} \in Z_{l}$ and $w_{j} \in W_{l}$ are two unmatched outputs of $N_{1}$ and $N_{2}$. Then, $z_{i}$ matches $w_{j}$ only if $\operatorname{Sign}\left(z_{i}\right)=\operatorname{Sign}\left(w_{j}\right)$.

As indicated in Section 5.1, matching two I/Os during SAT search introduces new singleton cells. These new cells might change the support signature of the remaining unmatched I/Os (the ones in the supports of the recently matched inputs or outputs). According to Lemma 10, this change in the support signatures might preclude some I/Os from matching. Taking advantage of this lemma, we can prune the unpromising branches of the SAT tree in the remaining space of matches.

\subsection{Pruning Invalid Input Matches Using Symmetries}

Our SAT-tree can exploit the symmetries of inputs to prune 1) impossible output matches, and 2) symmetric portions of the search space. Since computing the input 
symmetries of a Boolean network is expensive, the techniques explained in this section may in some cases hamper the matching process.

Definition 14. Let $X=\left\{x_{1}, \ldots, x_{n}\right\}$ be an input set of a Boolean network $N$. Let $x_{i} \sim x_{j}\left(\operatorname{read} x_{i}\right.$ is symmetric to $\left.x_{j}\right)$ if and only if the functionality of $N$ stays invariant under an exchange of $x_{i}$ and $x_{j}$. This defines an equivalence relation on set $X$, i.e., $\sim$ partitions $X$ into a number of symmetry classes where each symmetry class contains all the inputs that are symmetric to each other. The partition resulting from $\sim$ is called the symmetry partition of $X$.

For multi-output functions, symmetries of inputs are reported independently for each output. In other words, each output defines its own symmetry partition on inputs. Complying with the notion of symmetry in Definition 14, for a multi-output function, $x_{i}$ is called symmetric to $x_{j}$ if 1) $x_{i}$ and $x_{j}$ have the same output support, i.e., $\operatorname{Supp}\left(x_{i}\right)=\operatorname{Supp}\left(x_{j}\right)$, and 2) $x_{i}$ and $x_{j}$ are symmetric in all the outputs in their support, i.e., $x_{i} \sim x_{j}$ for all outputs in $\operatorname{Supp}\left(x_{i}\right)$ (or equivalently $\operatorname{Supp}\left(x_{j}\right)$ ).

Symmetries of inputs can serve as a signature for matching outputs in our SATbased search. The following lemma explains the role of symmetries in detecting invalid output matches.

Lemma 11. Output $z_{i} \in Z_{l}$ (from $N_{1}$ ) matches output $w_{j} \in W_{l}$ (from $N_{2}$ ) only if symmetry partition of $z_{i}$ is isomorphic to the symmetry partition of $w_{j}$ for at least one ordering of symmetry classes.

Input symmetries can also be used to prune symmetric parts of the search space during SAT-based exploration. Specifically, assume that the miter of $N_{1}^{\prime \prime}$ and $N_{2}^{\prime \prime}$ from Section 5.1 is satisfiable, i.e., $x_{i}$ does not match $y_{j}$. Based on the notion of input symmetries, if $x_{i}$ does not match $y_{j}$, neither can it match another input in $Y_{l}$ that is symmetric to $y_{j}$. In other words, $x_{i}$ cannot match $y_{q} \in Y_{l}$, if $y_{j}$ and $y_{q}$ are symmetric.

In practice, the use of symmetries in Boolean matching encounters two major limitations: 1) finding symmetries of a large Boolean network usually takes a significant amount of time, 2) in a case where a Boolean network does not have much symmetry, a considerable amount of time can be wasted.

\subsection{A Heuristic for Matching Candidates}

In order to reduce the branching factor of our SAT-tree, we first match I/Os of smaller I/O clusters. Also, within one I/O cluster, we exploit the observability of the inputs and the controllability of the outputs, to make more accurate guesses in our SAT-based matching approach. Heuristically, the probability that two I/Os match is higher when their observability/controllability are similar. We observed that, in many designs, the observability of control signals is higher than that of data signals. Therefore, we first match control signals. This simple heuristic can greatly improve the runtime - experiments indicate that once control signals are matched, data signals can be matched quickly. 


\section{Empirical Validation}

We have implemented the proposed approach in $\mathrm{ABC}$ and we have experimentally evaluated its performance on a $2.67 \mathrm{GHz}$ Intel Xeon CPU running Windows Vista. Table 1 and Table 2 show the runtime of our algorithms on ITC' 99 benchmarks for P-equivalence and PP-equivalence checking problems, respectively. In these two tables, \#I is the number of inputs, \#O is the number of outputs and $|\mathrm{AIG}|$ is the number of nodes in the AIG of each circuit. The last four columns demonstrate the initialization time (computing I/O support variables, initially refining I/O cluster and refining based on I/O dependencies), simulation time, SAT time, and overall time for each testcase. In addition to the reported runtimes, (I\%) and (I\%,O\%) show the percentage of inputs and I/Os that are matched after each step. Note that, in these experiments, we did not perform refinement using minterm counts and unateness, and we did not account for input symmetries to prune our SAT-tree because these techniques appear less scalable than the ones reported in Table 1 and Table 2. Also note that for each testcase we generated 20 new circuits each falling into one of the two following categories: (1) permuting inputs for verifying P-equivalence (2) permuting both inputs and outputs for verifying PP-equivalence. The results given in Table 1 and Table 2 are the average results over all the generated testcases for each category. Furthermore, the AIGs of the new circuits are reconstructed using ABC's combinational synthesis commands to ensure that the new circuits are structurally different from the original ones.

Table 1 P-equivalence runtime (sec.) and percentage of matched inputs for ITC'99 benchmarks

\begin{tabular}{|c|c|c|c|c|c|c|c|}
\hline Circuit & $\# \mathrm{I}$ & $\# \mathrm{O}$ & $|\mathrm{AIG}|$ & Initialization & Simulation & SAT & Overall \\
\hline b01 & 6 & 7 & 48 & $(66 \%)$ & o $(100 \%)$ & o $(100 \%)$ & 0.30 \\
\hline b02 & 4 & 5 & 28 & $0.28 \quad(50 \%)$ & o $(100 \%)$ & 0 $(100 \%)$ & 0.28 \\
\hline b03 & 33 & 34 & 157 & 0.36 $(97 \%)$ & о $\quad(97 \%)$ & $\mathbf{0 . 0 4}(100 \%)$ & 0.40 \\
\hline b04 & 76 & 74 & 727 & $\mathbf{0 . 4 1}(64 \%)$ & $0.04(100 \%)$ & o $(100 \%)$ & 0.45 \\
\hline b05 & 34 & 70 & 998 & $\mathbf{0 . 5 2}(84 \%)$ & $\mathbf{0 . 0 2}(100 \%)$ & o $(100 \%)$ & 0.54 \\
\hline b06 & 10 & 15 & 55 & $\mathbf{0 . 3 7}(80 \%)$ & 0 $(100 \%)$ & o $(100 \%)$ & 0.37 \\
\hline b07 & 49 & 57 & 441 & $0.41 \quad(67 \%)$ & $\mathbf{0 . 0 1}(100 \%)$ & o $(100 \%)$ & 0.43 \\
\hline b08 & 29 & 25 & 175 & $0.36 \quad(90 \%)$ & o $(100 \%)$ & o $(100 \%)$ & 0.36 \\
\hline b09 & 28 & 29 & 170 & $(100 \%)$ & o $(100 \%)$ & o $(100 \%)$ & 0.40 \\
\hline b10 & 27 & 23 & 196 & $(85 \%)$ & o $(100 \%)$ & o $(100 \%)$ & 0.34 \\
\hline b11 & 37 & 37 & 764 & $(95 \%)$ & $0.01 \quad(100 \%)$ & o $(100 \%)$ & 0.41 \\
\hline b12 & 125 & 127 & 1072 & $0.38 \quad(60 \%)$ & $0.25 \quad(100 \%)$ & o $(100 \%)$ & 0.63 \\
\hline b13 & 62 & 63 & 353 & $0.38 \quad(71 \%)$ & 0.01 $(100 \%)$ & o $(100 \%)$ & 0.39 \\
\hline b14 & 276 & 299 & 10067 & $6.89 \quad(73 \%)$ & $3.29(100 \%)$ & o $(100 \%)$ & 10.18 \\
\hline b15 & 484 & 519 & 8887 & $14.26(57 \%)$ & $\mathbf{5 . 8 2}(100 \%)$ & o $(100 \%)$ & 20.08 \\
\hline b17 & 1451 & 1512 & 32290 & $246 \quad(63 \%)$ & $46.14(99 \%)$ & $1.41(100 \%)$ & 294 \\
\hline b18 & 3357 & 3343 & 74900 & $2840 \quad(69 \%)$ & $51.6 \quad(99 \%)$ & $2.96(100 \%)$ & 2895 \\
\hline b20 & 521 & 512 & 20195 & $\mathbf{5 2 . 8}(83 \%)$ & $2.23 \quad(100 \%)$ & 0.01 (100\%) & 55 \\
\hline b21 & 521 & 512 & 20540 & $\mathbf{5 2 . 8}(83 \%)$ & $(100 \%)$ & 0.01 (100\%) & 55 \\
\hline b22 & 766 & 757 & 29920 & $150 \quad(82 \%)$ & $3.85(100 \%)$ & $0.32 \quad(100 \%)$ & 154 \\
\hline
\end{tabular}


Table 2 PP-equivalence runtime (sec.) and percentage of matched I/Os for ITC'99 benchmarks

\begin{tabular}{llllllll}
\hline Circuit & $\# \mathrm{I}$ & $\# \mathrm{O}$ & $|\mathrm{AIG}|$ & Initialization & Simulation & SAT & Overall \\
\hline b01 & 6 & 7 & 48 & $\mathbf{0 . 3 7}(50 \%, 43 \%)$ & $\mathbf{0}(83 \%, 85 \%)$ & $\mathbf{0 . 0 2}(100 \%, 100 \%)$ & $\mathbf{0 . 3 9}$ \\
b02 & 4 & 5 & 28 & $\mathbf{0 . 2 8}(50 \%, 60 \%)$ & $\mathbf{0}(100 \%, 100 \%)$ & $\mathbf{0}(100 \%, 100 \%)$ & $\mathbf{0 . 2 8}$ \\
b03 & 33 & 34 & 157 & $\mathbf{0 . 3 8}(48 \%, 38 \%)$ & $\mathbf{0 . 0 1}(54 \%, 47 \%)$ & $\mathbf{0 . 4 3}(100 \%, 100 \%)$ & $\mathbf{0 . 8 2}$ \\
b04 & 76 & 74 & 727 & $\mathbf{0 . 3 7}(16 \%, 13 \%)$ & $\mathbf{0 . 1}(100 \%, 100 \%)$ & $\mathbf{0}(100 \%, 100 \%)$ & $\mathbf{0 . 4 7}$ \\
b05 & 34 & 70 & 998 & $\mathbf{0 . 5 1}(34 \%, 24 \%)$ & $\mathbf{0 . 0 3}(54 \%, 47 \%)$ & $\mathbf{0 . 3 3}(100 \%, 100 \%)$ & $\mathbf{0 . 8 7}$ \\
b06 & 10 & 15 & 55 & $\mathbf{0 . 3 9}(30 \%, 47 \%)$ & $\mathbf{0}(50 \%, 53 \%)$ & $\mathbf{0 . 0 4}(100 \%, 100 \%)$ & $\mathbf{0 . 4 3}$ \\
b07 & 49 & 57 & 441 & $\mathbf{0 . 4 3}(67 \%, 70 \%)$ & $\mathbf{0 . 0 3}(94 \%, 95 \%)$ & $\mathbf{0 . 1 9}(100 \%, 100 \%)$ & $\mathbf{0 . 6 5}$ \\
b08 & 29 & 25 & 175 & $\mathbf{0 . 4 1}(27 \%, 36 \%)$ & $\mathbf{0 . 1 2}(100 \%, 100 \%)$ & $\mathbf{0}(100 \%, 100 \%)$ & $\mathbf{0 . 5 3}$ \\
b09 & 28 & 29 & 170 & $\mathbf{0 . 4 1}(46 \%, 48 \%)$ & $\mathbf{0 . 0 1}(46 \%, 48 \%)$ & $\mathbf{0 . 2 0}(100 \%, 100 \%)$ & $\mathbf{0 . 6 2}$ \\
b10 & 27 & 23 & 196 & $\mathbf{0 . 3 7}(88 \%, 95 \%)$ & $\mathbf{0}(100 \%, 100 \%)$ & $\mathbf{0}(100 \%, 100 \%)$ & $\mathbf{0 . 3 7}$ \\
b11 & 37 & 37 & 764 & $\mathbf{0 . 4 1}(65 \%, 65 \%)$ & $\mathbf{0}(100 \%, 100 \%)$ & $\mathbf{0 . 0 2}(100 \%, 100 \%)$ & $\mathbf{0 . 4 3}$ \\
b12 & 125 & 127 & 1072 & $\mathbf{0 . 3 8}(21 \%, 25 \%)$ & $\mathbf{1 . 0 5}(41 \%, 41 \%)$ & - & $\mathbf{0 . 1 4}$ \\
b13 & 62 & 63 & 353 & $\mathbf{0 . 3 5}(43 \%, 50 \%)$ & $\mathbf{0 . 0 5}(97 \%, 97 \%)$ & $\mathbf{0 . 1 4}(100 \%, 100 \%)$ & $\mathbf{0 . 5 4}$ \\
b14 & 276 & 299 & 10067 & $\mathbf{7 . 9 9}(72 \%, 58 \%)$ & $\mathbf{3 . 8 9}(89 \%, 90 \%)$ & $\mathbf{2 7}(100 \%, 100 \%)$ & $\mathbf{3 8 . 8}$ \\
b15 & 484 & 519 & 8887 & $\mathbf{1 6 . 4 0}(62 \%, 67 \%)$ & $\mathbf{4 5 . 6}(94 \% 94, \%)$ & $\mathbf{6 . 3 0}(100 \%, 100 \%)$ & $\mathbf{6 8 . 3}$ \\
b17 & 1451 & 1512 & 32290 & $\mathbf{2 4 9}(62 \%, 65 \%)$ & $\mathbf{2 2 9}(94 \%, 94 \%)$ & $\mathbf{1 4 8}(100 \%, 100 \%)$ & $\mathbf{6 2 6}$ \\
b18 & 3357 & 3343 & 74900 & $\mathbf{2 8 6 2}(65 \%, 63 \%)$ & $\mathbf{5 3 0}(93 \%, 93 \%)$ & - & \\
b20 & 521 & 512 & 20195 & $\mathbf{5 3 . 3}(70 \%, 51 \%)$ & $\mathbf{1 3 . 8 2}(89 \%, 89 \%)$ & $\mathbf{1 4 6}(100 \%, 100 \%)$ & $\mathbf{2 1 3}$ \\
b21 & 521 & 512 & 20540 & $\mathbf{5 3 . 3}(70 \%, 51 \%)$ & $\mathbf{1 1 . 7 0}(89 \%, 89 \%)$ & $\mathbf{1 5 9}(100 \%, 100 \%)$ & $\mathbf{2 2 5}$ \\
b22 & 766 & 757 & 29920 & $\mathbf{1 5 1}(70 \%, 50 \%)$ & $\mathbf{2 6 . 2 8}(88 \%, 88 \%)$ & $\mathbf{4 7 3}(100 \%, 100 \%)$ & $\mathbf{6 5 0}$ \\
\hline
\end{tabular}

— indicates runtime $>5000 \mathrm{sec}$.

In the ITC' 99 benchmark suite, 18 circuits out of 20 have less than a thousand I/Os. Checking P-equivalence and PP-equivalence for 12 out of these 18 circuits takes less than a second. There is only one circuit (b12) for which our software cannot match I/Os in 5000 seconds. The reason is that, for b12, 1033 out of 7750 input pairs (13\%) are symmetric and since our implementation does not yet account for symmetries, our SAT-tree repeatedly searches symmetric branches that do not yield valid I/O matches. For b20, b21 and b22 and for b17 and b18 with more than a thousand I/Os, computing functional dependency is the bottleneck of the overall matching runtime. Note that checking PP-equivalence for b18 results in a very large SAT-tree that cannot be resolved within 5000 seconds, although our refinement techniques before invoking SAT find exact matches for 3123 out of 3357 inputs (93\%) and 3111 out of 3343 outputs (93\%).

The results in Table 1 and Table 2 assume that target circuits are equivalent. In contrast, Table 3 considers cases where input circuits produce different output values on at least some inputs. For this set of experiments, we construced 20 inequivalent circuits for each testcase, using one of the following rules:

1. Wrong signals: outputs of two random gates were swapped.

2. Wrong polarity: an inverter was randomly added or removed.

3. Wrong gate: functionality of one random gate was altered.

In Table 3, columns Init, Sim, and SAT demonstrate the number of testcases (out of 20) for which our algorithms were able to prove inequivalence during initialization, simulation, and SAT search phases, respectively. Also, column Time shows 
Table 3 P-equivalence and PP-equivalence runtime (sec.) for ITC'99 benchmarks when mismatch exists

\begin{tabular}{llllllllllll}
\hline Circuit & $\# \mathrm{I}$ & \#O & $|\mathrm{AIG}|$ & \multicolumn{3}{c}{ P-equivalence } & \multicolumn{3}{c}{ PP-equivalence } \\
& & & & Init & Sim & SAT & Time & Init & Sim & SAT & Time \\
\hline b01 & 6 & 7 & 48 & 4 & 2 & 14 & $\mathbf{0 . 3 0}$ & 1 & 13 & 6 & $\mathbf{0 . 4 9}$ \\
b02 & 4 & 5 & 28 & 0 & 10 & 10 & $\mathbf{0 . 2 7}$ & 2 & 12 & 6 & $\mathbf{0 . 3 3}$ \\
b03 & 33 & 34 & 157 & 9 & 0 & 11 & $\mathbf{0 . 3 5}$ & 10 & 7 & 3 & $\mathbf{0 . 4 5}$ \\
b04 & 76 & 74 & 727 & 8 & 2 & 10 & $\mathbf{0 . 4 2}$ & 13 & 4 & 3 & $\mathbf{0 . 3 9}$ \\
b05 & 34 & 70 & 998 & 7 & 0 & 13 & $\mathbf{0 . 5 3}$ & 6 & 10 & 4 & $\mathbf{0 . 7 0}$ \\
b06 & 10 & 15 & 55 & 3 & 3 & 14 & $\mathbf{0 . 3 1}$ & 14 & 5 & 1 & $\mathbf{0 . 4 6}$ \\
b07 & 49 & 57 & 441 & 10 & 0 & 10 & $\mathbf{0 . 4 3}$ & 15 & 1 & 4 & $\mathbf{0 . 7 1}$ \\
b08 & 29 & 25 & 175 & 9 & 2 & 9 & $\mathbf{0 . 3 6}$ & 12 & 6 & 2 & $\mathbf{0 . 4 6}$ \\
b09 & 28 & 29 & 170 & 4 & 1 & 15 & $\mathbf{0 . 4 0}$ & 10 & 4 & 6 & $\mathbf{0 . 4 5}$ \\
b10 & 27 & 23 & 196 & 10 & 5 & 5 & $\mathbf{0 . 3 3}$ & 11 & 3 & 6 & $\mathbf{0 . 3 1}$ \\
b11 & 37 & 37 & 764 & 5 & 0 & 15 & $\mathbf{0 . 4 0}$ & 10 & 2 & 8 & $\mathbf{0 . 5 3}$ \\
b12 & 125 & 127 & 1072 & 6 & 10 & 4 & $\mathbf{0 . 4 5}$ & 10 & 8 & 2 & $\mathbf{3 . 5}$ \\
b13 & 62 & 63 & 353 & 6 & 9 & 5 & $\mathbf{0 . 3 8}$ & 7 & 7 & 6 & $\mathbf{0 . 5 5}$ \\
b14 & 276 & 299 & 10067 & 3 & 0 & 17 & $\mathbf{9 . 8 9}$ & 10 & 3 & 7 & $\mathbf{1 0 . 6 5}$ \\
b15 & 484 & 519 & 8887 & 4 & 2 & 14 & $\mathbf{2 0 . 0 3}$ & 8 & 4 & 8 & $\mathbf{3 8 . 2}$ \\
b17 & 1451 & 1512 & 32290 & 11 & 0 & 9 & $\mathbf{2 6 0}$ & 3 & 7 & 10 & $\mathbf{3 7 3}$ \\
b18 & 3357 & 3343 & 74900 & 2 & 0 & 18 & $\mathbf{2 8 6 4}$ & 0 & 9 & 11 & $-{ }^{2}$ \\
b20 & 521 & 512 & 20195 & 7 & 0 & 13 & $\mathbf{5 4}$ & 1 & 4 & 15 & $\mathbf{7 5 . 4}$ \\
b21 & 521 & 512 & 20540 & 2 & 0 & 18 & $\mathbf{5 4}$ & 5 & 11 & 4 & $\mathbf{5 9 . 4}$ \\
b22 & 766 & 757 & 29920 & 7 & 1 & 12 & $\mathbf{1 5 4}$ & 0 & 4 & 16 & $\mathbf{1 8 1}$ \\
\hline
\end{tabular}

— indicates runtime $>5000 \mathrm{sec}$.

${ }^{a}$ The average runtime excluding instances requiring SAT was $2902 \mathrm{sec}$.

the average runtime of our matcher for the P-equivalence and PP-equivalence problems. According to the results, our matcher resorts to SAT-solving in $45 \%$ of the testcases which suggests that many of our instances are not particularly easy. Moreover, calling SAT is due to the fact that our mismatched instances were all generated with minimal changes to the original circuits. Note that, even in the case of a slight mismatch, our signature-based techniques alone could effectively discover inequivalence for $55 \%$ of testcases. Furthermore, comparing the results in Table 2 and Table 3 , PP-equivalence checking is up to 4 times faster when mismatch exists. In particular, for b12, our matcher could confirm inequivalence in less than 5 seconds, even when SAT-solving was invoked. The reason is that in the case of a mismatch, our SAT-tree usually encounters invalid I/O matches early in the tree, which results in a vast pruning in the space of invalid matches.

In order to compare our work to that in [17], we have tested our algorithms on circuits from [17] that have more than 150 inputs. Results are listed in Table 4. For the results reported from [17], Orig, Unate and +Symm respectively show the runtime when no functional property is used, only functional unatness is used and, both unateness and symmetries are used. Note that experiments reported in [17] used $3 \mathrm{GHz}$ Intel CPUs, while our runs were on a $2.67 \mathrm{GHz}$ Intel CPU. To make the numerical comparisons entirely fair, our runtimes would need to be multiplied by 0.89 . However, we omit this step, since our raw runtimes are already superior in 
Table 4 P-equivalence runtime (sec.) compared to runtime (sec.) from [17]

\begin{tabular}{llllllllll}
\hline Circuit & $\# \mathrm{II}$ & \#O & \multicolumn{3}{c}{ P-equivalence Runtime (sec.) } & \multicolumn{2}{c}{ CPU Time (sec.) in [17] } \\
& & & Init & Sim & SAT & Overall & Orig & +Unate + Sym \\
\hline C2670 & 233 & 140 & 0.14 & 1.18 & - & - & - & - & $\mathbf{7 . 9 6}$ \\
C5315 & 178 & 123 & 0.33 & 0.11 & 0.06 & $\mathbf{0 . 5}$ & $\mathbf{6 . 3 1}$ & $\mathbf{2 . 8 6}$ & $\mathbf{3 . 2 9}$ \\
C7552 & 207 & 108 & 0.51 & 3.76 & 4.83 & $\mathbf{9 . 1 0}$ & - & - & $\mathbf{1 4 . 5 6}$ \\
des & 256 & 245 & 0.38 & 0.07 & 0 & $\mathbf{0 . 4 5}$ & $\mathbf{1 0 . 2 1}$ & $\mathbf{0 . 2 5}$ & $\mathbf{2 . 3 3}$ \\
i10 & 257 & 224 & 0.43 & 1.03 & 1.23 & $\mathbf{2 . 6 9}$ & $\mathbf{2 5 . 6 3}$ & $\mathbf{1 5 . 1 6}$ & $\mathbf{1 7 . 5 6}$ \\
i2 & 201 & 1 & 0.34 & 0.28 & - & - & - & - & $\mathbf{1 . 0 2}$ \\
i4 & 192 & 6 & 0.31 & 0.27 & - & - & - & - & $\mathbf{0 . 2 2}$ \\
i7 & 199 & 67 & 0.36 & 0.18 & 0 & $\mathbf{0 . 5 4}$ & $\mathbf{0 . 8 2}$ & $\mathbf{0 . 0 4}$ & $\mathbf{0 . 1 9}$ \\
pair & 173 & 137 & 0.32 & 0.14 & 0 & $\mathbf{0 . 4 6}$ & $\mathbf{0 . 8 4}$ & $\mathbf{0 . 6 4}$ & $\mathbf{2 . 4 4}$ \\
s3384 & 226 & 209 & 0.10 & 0.25 & 0.47 & $\mathbf{0 . 8 2}$ & $\mathbf{4 . 7 9}$ & $\mathbf{2 . 1 4}$ & $\mathbf{4 . 0 2}$ \\
s5378 & 199 & 213 & 0.11 & 0.53 & 0.63 & $\mathbf{1 . 2 7}$ & $\mathbf{1 . 3 1}$ & $\mathbf{3 . 3 8}$ & $\mathbf{2 . 4 2}$ \\
s9234 & 247 & 250 & 3.11 & 0.53 & 2.85 & $\mathbf{6 . 4 9}$ & $\mathbf{3 . 4 1}$ & $\mathbf{5 . 8 4}$ & $\mathbf{7 . 8 2}$ \\
s38584 & 1464 & 1730 & 58 & 1.66 & 1.54 & $\mathbf{6 1}$ & $\mathbf{7 6}$ & $\mathbf{2 1 0}$ & $\mathbf{4 5 8}$ \\
s38417 & 1664 & 1742 & 50 & 9.46 & 30.9 & $\mathbf{9 0}$ & $\mathbf{9 1}$ & $\mathbf{3 2 4}$ & $\mathbf{9 9 9}$ \\
\hline
\end{tabular}

- indicates runtime $>5000 \mathrm{sec}$.

many cases. According to Table 4, our matching algorithm times out in $5000 \mathrm{sec}-$ onds on C2670, i2 and i4. This is again due to the symmetries that are present in the inputs of these circuits. Note that the approach in [17] cannot solve these three circuits without symmetry search, either. For some other circuits, such as C7552, our approach verifies P-equivalence in less than 10 seconds but the approach in [17] cannot find a match without invoking symmetry finder. It is also evident from the results that checking P-equivalence for very large circuits, such as s38584 and s38417, is 3.5-11 times slower when symmetry finding and unateness calculations are performed during Boolean matching. This confirms our intuition that symmetry and unateness are not essential to Boolean matching in many practical cases, although they may occasionally be beneficial.

\section{Chapter Summary}

In this chapter, we proposed techniques for solving large-scale PP-equivalence checking problem. Our approach integrates graph-based, simulation driven and SAT-based techniques to efficiently solve the problem. Graph-based techniques limit dependencies between inputs and outputs and are particularly useful with word-level arithmetic circuits. Simulation quickly discovers inputs on which inequivalent circuits differ. Equivalences are confirmed by invoking SAT, and these invocations are combined with branching on possible matches. Empirical validation of our approach on available benchmarks confirms its scalability to circuits with thousands of inputs and outputs. Future advances in Boolean matching, as well as many existing techniques, can also be incorporated into our framework to improve its scalability. 


\section{References}

1. Abdollahi, A.: Signature based boolean matching in the presence of don't cares. In: DAC '08: Proceedings of the 45th annual Design Automation Conference, pp. 642-647. ACM, New York, NY, USA (2008). DOI http://doi.acm.org/10.1145/1391469.1391635

2. Abdollahi, A., Pedram, M.: A new canonical form for fast boolean matching in logic synthesis and verification. In: DAC '05: Proceedings of the 42nd annual Design Automation Conference, pp. 379-384. ACM, New York, NY, USA (2005). DOI http://doi.acm.org/10.1145/1065579.1065681

3. Agosta, G., Bruschi, F., Pelosi, G., Sciuto, D.: A unified approach to canonical formbased boolean matching. In: DAC '07: Proceedings of the 44th annual Design Automation Conference, pp. 841-846. ACM, New York, NY, USA (2007). DOI http://doi.acm.org/10.1145/1278480.1278689

4. Benini, L., Micheli, G.D.: A survey of boolean matching techniques for library binding. ACM Transactions on Design Automation of Electronic Systems 2, 193-226 (1997)

5. Chai, D., Kuehlmann, A.: Building a better boolean matcher and symmetry detector. In: DATE '06: Proceedings of the conference on Design, automation and test in Europe, pp. 1079-1084. European Design and Automation Association, 3001 Leuven, Belgium, Belgium (2006)

6. Davis, M., Logemann, G., Loveland, D.: A machine program for theorem-proving. Commun. ACM 5(7), 394-397 (1962). DOI http://doi.acm.org/10.1145/368273.368557

7. Davis, M., Putnam, H.: A computing procedure for quantification theory. J. ACM 7(3), 201215 (1960). DOI http://doi.acm.org/10.1145/321033.321034

8. Eén, N., Sörensson, N.: An extensible SAT-solver. In: E. Giunchiglia, A. Tacchella (eds.) SAT, Lecture Notes in Computer Science, vol. 2919, pp. 502-518. Springer (2003)

9. Goering, R.: Xilinx ISE handles incremental changes. http://www.eetimes.com/showArticle.jhtml?articleID=196901122 (2007)

10. Krishnaswamy, S., Ren, H., Modi, N., Puri, R.: Deltasyn: an efficient logic difference optimizer for ECO synthesis. In: ICCAD '09: Proceedings of the 2009 International Conference on Computer-Aided Design, pp. 789-796. ACM, New York, NY, USA (2009). DOI http://doi.acm.org/10.1145/1687399.1687546

11. Lee, C.C., Jiang, J.H.R., Huang, C.Y.R., Mishchenko, A.: Scalable exploration of functional dependency by interpolation and incremental SAT solving. In: ICCAD '07: Proceedings of the 2007 IEEE/ACM international conference on Computer-aided design, pp. 227-233. IEEE Press, Piscataway, NJ, USA (2007)

12. Mishchenko, A.: Logic synthesis and verification group. ABC: A system for sequential synthesis and verification, release 70930. http://www.eecs.berkeley.edu/ãlanmi/abc/

13. Mishchenko, A., Chatterjee, S., Brayton, R.: FRAIGs: A unifying representation for logic synthesis and verification. Tech. rep., UC Berekeley (2005)

14. Mishchenko, A., Chatterjee, S., Brayton, R., Een, N.: Improvements to combinational equivalence checking. In: ICCAD '06: Proceedings of the 2006 IEEE/ACM international conference on Computer-aided design, pp. 836-843. ACM, New York, NY, USA (2006). DOI http://doi.acm.org/10.1145/1233501.1233679

15. Ray, S., Mishchenko, A., Brayton, R.: Incremental sequential equivalence checking and subgraph isomorphism. In: Proc. of the Intl. Workshop on Logic Synthesis, pp. 37-42 (2009)

16. S. Nocco, S.Q.: A probabilistic and approximated approach to circuit-based formal verification. Journal of Satisfiability, Boolean Modeling and Computation 5, 111-132 (2008)

17. Wang, K.H., Chan, C.M., Liu, J.C.: Simulation and SAT-based boolean matching for large boolean networks. In: DAC '09: Proceedings of the 46th Annual Design Automation Conference, pp. 396-401. ACM, New York, NY, USA (2009). DOI http://doi.acm.org/10.1145/1629911.1630016 



\section{Index}

And-Inverter graph (AIG), 4

binate, 4

Boolean matching, 1

Boolean satisfiability, 5

canonical form based matching, 2

complete partitions, 4

consistent input vectors, 10

controllability, 11

enhanced CEC, 2

generalized Boolean matching, 1

I/O cluster, 4

I/O cluster mapping, 6

I/O degree, 7

I/O mapping, 6

I/O signature, 2

I/O support, 7

I/O support signature, 9

I/O support variable, 7

isomorphic partitions, 4

miter, 5

negative cofactor, 4 negative unate, 4

observability, 11

ordered partition, 4

output vector, 10

partition, 4

PNPN-equivalence checking, 2

positive cofactor, 4

positive unate, 4

PP-equivalence checking, 2, 6

proper input vector, 10

refinement, 6

SAT, 5

SAT-tree, 12

satisfiable assignment, 5

signature-based matching, 2

smallest matching sub-circuits, 13

structural hashing, 5

symmetry, 16

symmetry class, 16

symmetry partition, 16

trivial proper input vector, 10

unsatisfiable assignment, 5 